\title{
Redescobrindo a lei empírica de Hubble em sala de aula
}

\author{
V R Peçanha da Rocha ${ }^{1}$, A C Tort ${ }^{*}$ \\ ${ }^{1}$ Universidade Federal do Rio de Janeiro, Instituto de Física, Mestrado Nacional Prossional em Ensino de Física, Rio de \\ Janeiro, RJ, Brasil
}

Recebido em 09 de Outubro, 2018. Revisado em 30 de Janeiro, 2019. Aceito em 28 de Fevereiro, 2019.

\begin{abstract}
O século 20 é marcado por duas grandes descobertas cosmológicas: a expansão do Universo, e a medida da radiação cósmica de fundo correspondente a uma temperatura de $T=2.275 \mathrm{~K}$. Esta última, conjuntamente com a predição correta da abundância dos elementos formam os pilares que sustentam o modelo cosmológico atual ou modelo cosmológico padrão. Neste trabalho propomos a redescoberta da lei empírica de Hubble em sala de aula por meio de atividades práticas que promovem a participação ativa dos alunos. A lei empírica de Hubble é o ponto de partida da concepção de que nosso universo está em expansão,
\end{abstract}

Palavras-chave: lei de Hubble, deslocamento para o vermelho, expansão do universo.

The 20th century witnessed two great cosmological discoveries: The Cosmic Background Radiation corresponding to a black body temperature $T=2.275 \mathrm{~K}$, and the expansion of the universe expressed by the cosmological version of Hubble's empirical law of 1929. In this paper we propose a pedagogical reconstruction in class of the original Hubble's law with a hands-on approach making use of modern resources freely available on the web.

Keywords: Hubble's law, redshift, expansion of the universe.

\section{Introdução}

O século 20 é marcado por duas grandes descobertas cosmológicas: a expansão do Universo, e a medida da radiação cósmica de fundo. Esta última, conjuntamente com a predição correta da abundância dos elementos formam os pilares que sustentam o modelo cosmológico atual ou modelo padrão. Neste trabalho propomos a reconstrução de modo didático da descoberta da lei de Hubble que estendida e interpretada à luz dos modelos cosmológicas apropriados tornou-se evidência experimental de que as galáxias estão em movimento de recessão em relação ao Sol, ou melhor: em relação ao sistema de coordenadas heliocêntricas.

O presente trabalho está dividido em duas partes: na primeira parte, seção 2, discutimos as ferramentas que são utilizadas para estudar a lei de Hubble e suas consequências. Na segunda parte, seções 3, 4, procuramos prover o professor com uma bagagem conceitual mínima que lhe permita discutir com seus alunos a lei de Hubble original e sua versão cosmológica. Na terceira parte, seção 5 , sugerimos atividades práticas para a sala de aula.

\section{As ferramentas: espectroscopia e efeito Doppler relativístico}

Na primeira década do século 20, o uso da espectrometria e do efeito Doppler para a luz era um procedimento

*Endereço de correspondência: tort@if.ufrj.br bem estabelecido para a medição da velocidade radial do movimento próprio das estrelas [1] em relação ao Sol ( sistema heliocêntrico) - veja a Figura 1. Uma estrela é uma fornalha nuclear que produz energia continuamente pelo processo de fusão nuclear, mas o padrão da radiação emitida que observamos provém das suas camadas superiores. Este padrão é a assinatura química da estrela. Se a estrela não se move em relação observador, o padrão é o mesmo que seria obtido em uma laboratório terrestre, mas se a estrela se move, o padrão sofrerá um deslocamento - veja a Figura 2. Se a estrela aproxima-se do observador, o espectro tende para frequências mais altas ou, no jargão dos espectroscopistas, sofre um deslocamento para o azul. Se a estrela afasta-se do observador, o espectro tende para frequências mais baixas, isto é: sofre um deslocamento para o vermelho. Estes deslocamentos são quantificados por meio de um parâmetro convenientemente definido, o parâmetro $z$ ou deslocamento para $o$ vermelho.

Este parâmetro pode ser definido de dois modos equivalantes. Um modo utiliza a frequência e o outro o comprimento de onda, embora seja este último o que é medido pelos astrofísicos.

UTILIZANDO A FREQUÊNCIA:

$$
z=\frac{f_{\mathrm{E}}-f_{\mathrm{R}}}{f_{\mathrm{R}}}
$$

onde $\nu_{\mathrm{E}}$ é a frequência da radiação emitida pela fonte emissora (estrela ou galáxia) e $\nu_{\mathrm{R}}$ é frequência da radiação registrada no receptor. 


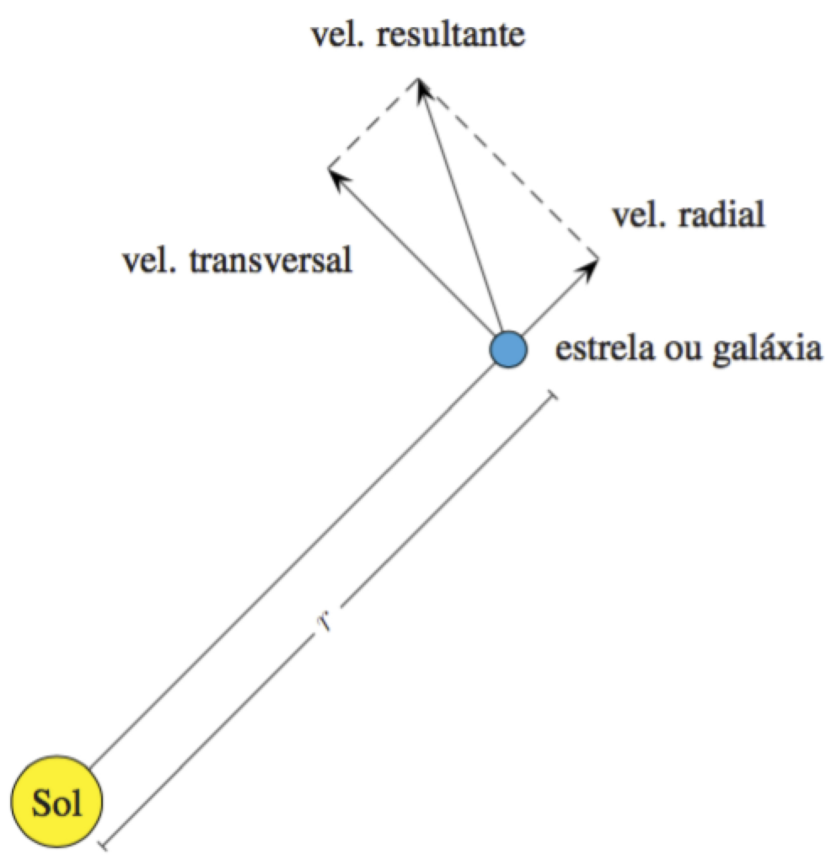

Figura 1: Movimento próprio de uma estrela ou galáxia em relação Sol.

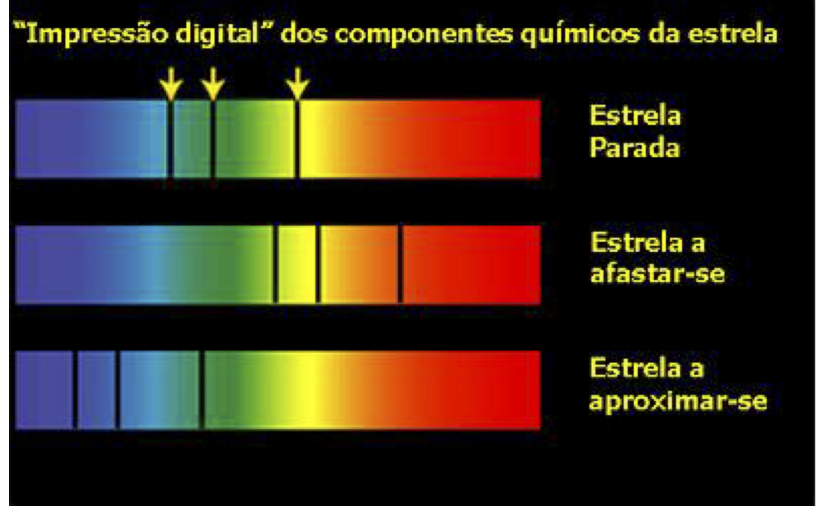

Figura 2: Espectro de absorção de uma estrela (linhas escuras) em três situações cinemáticas diferentes. (Imagem Wikipedia Commons)

\section{UTILIZANDO O COMPRIMENTO DE ONDA:}

$$
z=\frac{\lambda_{\mathrm{R}}-\lambda_{\mathrm{E}}}{\lambda_{\mathrm{E}}}
$$

Combinando o parâmetro $z$ com as equações do efeito Doppler relativístico podemos obter a velocidade radial da fonte em relação ao receptor $R$ (ou observador). Tenhamos em mente que é o comprimento de onda a grandeza física medida pelos astrofísicos. Para velocidades radiais baixas em comparação com a velocidade da luz $c$, a relação entre a velocidade da estrela/galáxia e o parâmetro $z$ se escreve:

$$
V_{r}=c z,
$$

onde $V_{r}$ é a componente radial da velocidade própria heliocêntrica da estrela (ou galáxia). Isto significa que a velocidade radial medida pelo observador terrestre deve ser corrigida levando em conta a velocidade do Sol em relação à um grupo de estrelas fixas.

\section{Nebulosas espirais, debris de estrelas, sistemas planetários em formação ou universos-ilha?}

Uma vez definidas as ferramentas que serão utilizadas para redescobrir a lei empírica de Hubble, convém ter uma idéia do contexto em que ela surge.

Ao final do século do 19 e princípios do século 20, um tema importante dominava as discussões astronômicas. O problema, originado no século anterior, era a natureza das nebulosas espirais como eram então chamadas as atuais galáxias espirais. Seriam elas o resultado da geração de novas estrelas ou sistemas planetários ou seriam outros conjuntos de estrelas similares à nossa Via Láctea? A questão era de difícil solução, pois os telescópios da época não eram capazes de distinguir estruturas no interior dessas nebulosas. Enquanto William Herschel (1738-1822), o grande astronômo britânico do século 18, acreditava que nossa galáxia era única no universo, o filósofo alemão Immanuel Kant (1724-1804), seu contemporâneo, acreditava que as nebulosas seriam coleções de estrelas, como a Via Láctea. Kant denominava tais coleções de estrelas "mundos-ilha" ou universos-ilha. A questão ficou resolvida apenas quando os telescópios foram equipados com espectrógrafos e as linhas espectrais registradas nas placas fotográficas analisadas à luz do efeito Doppler. O primeiro a fazê-lo foi o astronônomo inglês William Huggins (1824-1910). Em 1868, Huggins apontou seu telescópio para a estrela Sirius e ao analisar o seu espectro verificou um pequeno deslocamento das linhas espectrais que interpretado como efeito Doppler mostrava que na época, Sirius 11 afastava-se do observador com uma velocidade da ordem de $50 \mathrm{~km} / \mathrm{s}$ [1.

Em setembro de 1912, o astrônomo americano Vesto Melvin Slipher (1875-1969) obteve o primeiro espectrograma de boa qualidade da galáxia, então nebulosa, de Andrômeda (M31 ou NGC 224) [1,2]. Um pouco mais tarde, em janeiro de 1913, Slipher obteve quatro placas adicionais nas quais as linhas espectrais estavam claramente registradas. Analisando as linhas espectrais, Slipher conclui que elas estavam ligeiramente deslocadas para o azul. Andrômeda estava aproximando-se da Via Láctea com uma velocidade radial de $300 \mathrm{~km} / \mathrm{s}$, um valor dez maior do que a velocidade radial de uma estrela! [1,2]. Por volta de 1922, Slipher tinha medidas de velocidades radiais de 41 nebulosas espirais. Estes dados foram publicados por Eddington [3, veja a Figura 3. As velocidades radiais dessas nebulosas são da ordem de $10^{2} \mathrm{~km} / \mathrm{s}$. Como já mencionado, para estrelas, a velocidade radial típica é da ordem de $10 \mathrm{~km} / \mathrm{s}$. Mas, observemos também que

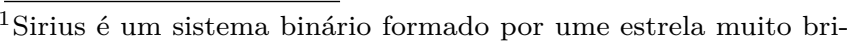
lhante, Sirius A, e uma anã branca, Sirius B.
} 


\begin{tabular}{|c|c|c|c|c|c|c|c|}
\hline \multirow[b]{2}{*}{ s, G.c. } & \multicolumn{6}{|c|}{$\begin{array}{c}\text { Radial Velocities of Spiral Nebulae } \\
+ \text { iudicates receding, }- \text { approaching }\end{array}$} & \multirow[b]{2}{*}{$\begin{array}{c}\text { Rad. Vel. } \\
\text { km. per sec }\end{array}$} \\
\hline & $\begin{array}{l}\text { 1., A. } \\
h \quad m\end{array}$ & Dee. & $\begin{array}{l}\text { Rad. Vel. } \\
\text { km. per sec. }\end{array}$ & s.c.c. & $\begin{array}{l}\text { r. A. } \\
h \quad m\end{array}$ & Dec. & \\
\hline 221 & 038 & +4026 & -300 & $4151^{*}$ & 126 & +3951 & +980 \\
\hline $224 *$ & 038 & +4050 & -300 & 4214 & 1212 & +3646 & +300 \\
\hline $278+$ & 047 & +477 & +650 & 4258 & 1215 & +4745 & +500 \\
\hline 404 & 15 & +3517 & $-\quad 25$ & $4382+$ & 1221 & +1839 & +500 \\
\hline $584+$ & 127 & -717 & +1800 & 4449 & 1224 & +4432 & +200 \\
\hline $598^{*}$ & 129 & +3015 & -260 & 4472 & 1225 & +827 & +850 \\
\hline 936 & 224 & -131 & +1300 & $4486 t$ & 1227 & +1250 & +800 \\
\hline 1023 & 235 & +3843 & +300 & 4526 & 1230 & +89 & +580 \\
\hline $1068 *$ & 239 & -021 & +1120 & $4565 t$ & 1232 & +2626 & +1100 \\
\hline 2683 & 848 & +3343 & +400 & $4594^{*}$ & 1236 & -1111 & +1100 \\
\hline $2841+$ & 916 & +5119 & +600 & 4649 & 1240 & +120 & +1090 \\
\hline 3031 & 949 & +6927 & $-\quad 30$ & 4736 & 1247 & +4133 & +290 \\
\hline 3034 & 949 & +705 & +290 & 4826 & 1253 & +227 & +150 \\
\hline $3115+$ & 101 & -720 & +600 & 5005 & 137 & +3729 & +900 \\
\hline 3368 & 1042 & +1214 & +940 & 5055 & 1312 & +4237 & +450 \\
\hline $3379^{*}$ & 1043 & +139 & +780 & 5194 & 1326 & +4736 & +270 \\
\hline $3489+$ & 1056 & +1420 & +600 & $5195+$ & 1327 & +4741 & +240 \\
\hline 3521 & 112 & +024 & +730 & $5236+$ & 1332 & $-29 \quad 27$ & +500 \\
\hline 3623 & 1115 & +1332 & +800 & 5866 & 154 & $+56 \quad 4$ & +650 \\
\hline 3627 & 1116 & +1326 & +650 & 7331 & $22 \quad 33$ & +3323 & +500 \\
\hline $4111+$ & 123 & +4331 & +800 & & & & \\
\hline
\end{tabular}

Figura 3: Velocidades radiais de 41 nebulosas espirais observadas por V. Slipher; uma velocidade negativa indica que a nebulosa (galáxia) aproxima-se do observador, positiva indica afastamento. NGC 221 é a galáxia espiral Andrômeda. Estes dados foram publicados por A. S. Eddington em [3].

alguns destes valores de velocidade radial são tão altos (da ordem de $10^{3}$ ) que podem ser interpretados como um indício de que pelo menos algumas dessas nebulosas não estão gravitacionalmente ligadas à Via Láctea.

A questão seria resolvida por Edwin Hubble (1889-1953) que se tornaria o astrônomo de maior prestígio da primeira metade do século 20. Hubble resolveu inicialmente o problema da medida da distância às nebulosas utilizando o método das Cefeidas descoberto por Henrietta Leavitt (1868-1921). Hubble localizou Cefeidas em três nebulosas e com isto foi capaz de calcular as respectivas distâncias. As distâncias calculadas mostraram que as nebulosas espirais localizavam-se muito além dos limites da Via Láctea.

\subsection{A lei empírica de Hubble}

Em 1929, como consequência de sua análise do movimento do Sol em relação às nebulosas - que hoje denominamos galáxias - Hubble chegou à conclusão que estas na maior parte dos casos afastavam-se do Sol com uma velocidade heliocêntrica radial linearmente proporcional à distância entre o Sol e as galáxias [4,5], isto é:

$$
V_{r}=K d,
$$

onde $K$ é uma constante e $V_{r}$ é a velocidade radial heliocêntrica, isto é: a velocidade radial da galáxia em relação Sol, obtida uma vez subtraídas a velocidade orbital da
Terra em torno do Sol, a velocidade de rotação da Terra e a velocidade da Terra em relação ao centro de massa do sistema Terra-Lua, uma correção cinemática que não discutiremos aqui - para uma abordagem didática veja [6]. Hoje em dia escrevemos a relação acima em uma forma ligeiramente diferente

$$
V_{r}=H_{0} d,
$$

onde $H_{0}$ é a constante de Hubble medida no tempo presente, pois hoje sabemos que a constante de Hubble não é realmente uma constante e varia no decurso do tempo cosmológico. Numericamente, o seu valor atual é de $H_{0}=100 h \mathrm{~km} \mathrm{~s}^{-1} \mathrm{Mpc}^{-1}$, onde $h$ é um fator de ajuste que está entre 0,5 e 1,0, logo, $50<H_{0}<100$ $\mathrm{km} \mathrm{s}^{-1} \mathrm{Mpc}^{-1}$. A unidade de distância empregada é o parsec $(\mathrm{pc})$ e $1 \mathrm{pc} \approx 3,2$ anos-luz; $1 \mathrm{Mpc}=10^{6} \times 1 \mathrm{pc}$.

Se $V / c \ll 1$, a equação (4) pode ser escrita na forma equivalente

$$
z c=H_{0} d,
$$

onde, como vimos antes, $z$ é o parâmetro que mede o deslocamento das raias espectrais, ou redshift. A Figura 4 mostra o famoso gráfico publicado no artigo de 1929. O gráfico mostra a relação entre a velocidade radial das nebulosas/galáxias levando em conta o movimento do Sol- a velocidade heliocêntrica - e a distância entre o observador e as nebulosas. As distâncias são medidas em 


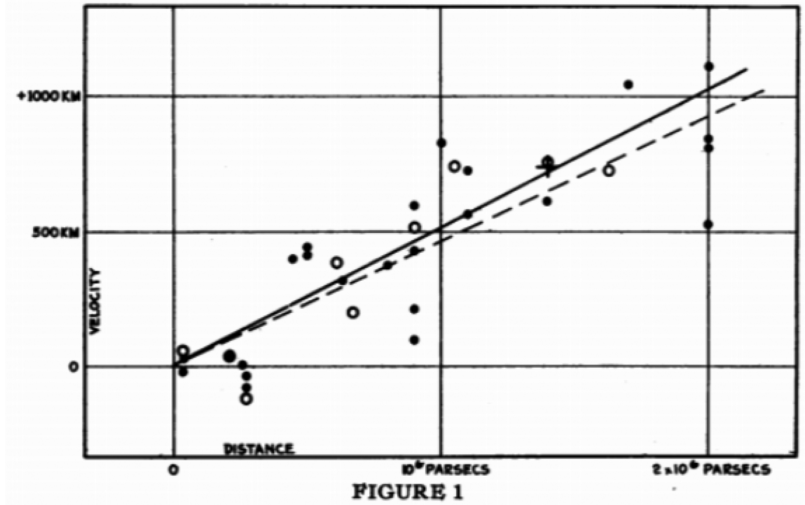

Velocity-Distance Relation among Extra-Galactic Nebulae.

Figura 4: Resultados publicados por Hubble em 1929 4].

parsecs $\times 10^{6}$. Observe que a unidade de velocidade está incorreta. Os círculos cheios • e a linha cheia representam nebulosas individuais; os círculos o e a linha tracejada representam nebulosas combinadas em grupos. A maior parte das distâncias foi determinada pelas estrelas mais brilhantes que pertencem a uma nebulosa/galáxia ou pela luminosidade das próprias nebulosas/galáxias. Há um erro sistemático na estimativa das distâncias de um fator sete [7] que, não obstante, não invalida a linearidade expressa pela equações (4) e (5). O valor da constante $H_{0}$ encontrado por Hubble é $465 \mathrm{~km} \mathrm{~s}^{-1} \mathrm{Mpc}^{-1}$.

O inverso da constante de Hubble tem dimensões de tempo e é definido como o tempo de Hubble, $\mathrm{T}_{\mathrm{H}}$, seu valor para $H_{0}=70 \mathrm{~km} \mathrm{~s}^{-1} \mathrm{Mpc}^{-1}$ é

$$
\mathrm{T}_{\mathrm{H}}=\frac{1}{H_{0}} \approx 14 \text { bilhões de anos. }
$$

O tempo de Hubble fixa uma escala de tempo na qual a expansão do universo deve ser levada em conta, por exemplo, comparada com $\mathrm{T}_{\mathrm{H}}$, a expansão do universo no decorrer de, digamos, 12000 de história humana é muito pequena, mas comparada com a idade estimada da Terra, aproximadamente 5 bilhões de anos, pode ser signiificativa. Os resultados de Hubble são uma indicação preliminar que as galáxias em sua maioria estão afastando-se do observador e não que o universo está em expansão.

Em 1931, Hubble e Humason publicaram novos resultados estendendo o domínio das distâncias de $6 \mathrm{Mpc}$ para $30 \mathrm{Mpc}$. [8]. O resultado é o gráfico à direita da Figura 5. Observe que a região explorada por Hubble no artigo de 1929 é relativamente pequena.

\section{A versão cosmológica da lei Hubble}

Na forma expressa pela equação (5), a lei de Hubble, também conhecida como a relação entre o redshift e a distância, deve ser considerada como uma relação empírica, isto é: resultado de observações diretas dos fenômenos valendo para $0<z<0,1$ 12].

A relação empírica entre a velocidade de afastamento de uma galáxia e a sua distância ao observador não deve ser confundida com a relação mais geral obtida a partir do modelo cosmológico presentemente aceito que é dada por:

$$
V_{r}=H(t) \ell_{P}
$$

onde $\ell_{P}$ é a distância própria entre o observador e a galáxia, e a "constante" de Hubble é função do tempo cosmológico, mas não do espaço. A distância própria é o valor que mediríamos com nossas réguas se pudéssemos congelar o universo e medir a distância entre dois pontos. A equação (6) define o fluxo de Hubble e é o resultado de trabalhos teóricos que começaram com o físico russo Alexander Friedmann (1888-1925) por volta de 1922 9] e prosseguiram com Georges Lemaître (1894-1966), Arthur Eddington (1882-1944), Richard Tolman (1881-1948), Howard Robertson (1903-1961), Edward Milne (1896-1950) Arthur Walker (1909-2001) nos anos 1930 [10,11]. A equação (6) é consequência do modelo cosmológico adotado que
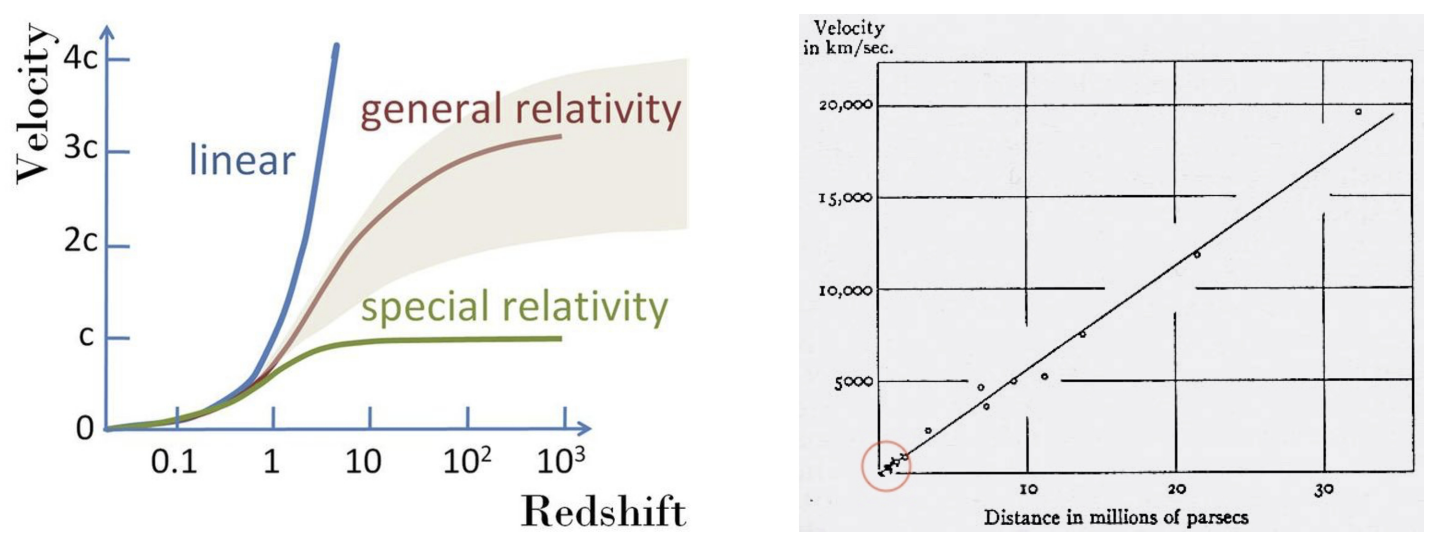

Figura 5: À esquerda, o gráfico mostra o deslocamento para o vermelho no eixo das abcissas e a velocidade no eixo das ordenadas. Note que a escala no eixo das abcissas é geométrica. As limitações da lei empírica de Hubble: $0<z<0,1$, são evidentes. À direita, o gráfico de 1931 de Humason e Hubble [8]: nas abcissas temos as distâncias em milhões de parsecs ou Mpc e nas ordenadas, a velocidade em km/s. O círculo indica a região explorada por Hubble no artigo de 1929. (Imagens Wikipedia Commons). 
considera nosso universo homogêneo, isotrópico. Com este modelo, é possível mostrar que a distância própria entre o observador e uma galáxia pode ser escrita na forma 12,13

$$
\ell_{P}=a(t) r
$$

onde $r$ é a distância comóvel entre o observador e uma galáxia ou entre duas galáxias, veja a Figura 6 . A função $a(t)$ é o fator de escala e no instante presente $t_{0}$ tomado como referência, vale a unidade, isto é: $a\left(t_{0}\right)=1$. A velocidade de afastamento é definida por

$$
V=\frac{d \ell_{P}}{d t}=\frac{d a(t)}{d t} r=\frac{d a(t) / d t}{a(t)} a(t) r=H(t) \ell_{P},
$$

onde definimos a "constante de Hubble" por:

$$
H(t)=\frac{1}{a(t)} \frac{d a(t)}{d t} .
$$

A constante empírica de Hubble corresponde a

$$
\left.H_{0}=\frac{1}{a(t)} \frac{d a(t)}{d t}\right]_{t=t_{0}},
$$

onde $t_{0}$ é a época atual. Embora a lei empírica de Hubble nos indique que as galáxias estão em movimento de recessão é a equação (6) que permite-nos afirmar que o universo está em expansão. A Figura 5 ilustra os dois tipos de deslocamento para o vermelho de que estamos falando. A linha azul corresponde à aproximação linear, $V_{r} \approx z c$, válida para $0<z<1$, a linha verde corresponde à fórmula exata da relatividade restrita para o parâmetro $z$ :

$$
\frac{V_{r}}{c}=\frac{z^{2}+2 z}{z^{2}+2 z+2} .
$$

Observe que para $z \rightarrow 0, V_{r} / c \rightarrow z$. A linha marrom corresponde ao fluxo de Hubble ou redshift cosmológico e depende dos aspectos do modelo cosmológico que determinam o fator de escala $a(t)$ que por sua vez determina $H(t)$. Observe que a escala no eixo das abcissas é geométrica e por esta razão as curvas mostradas nos parecem não-familiares.

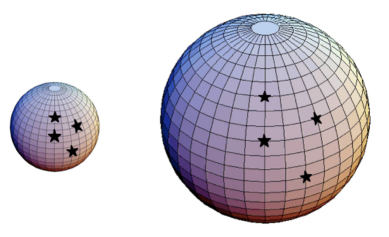

Uniform expansion yields the Hubble Law

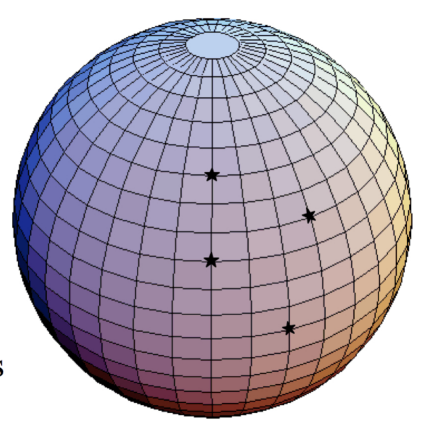

Figura 6: Uma expansão uniforme leva à lei de Hubble. (Imagem Wikipedia Commons)
Há um terceiro tipo de redshift, o gravitacional, que não é importante para nossos propósitos aqui. As distinções entre os redshifts são discutidas com detalhes nas referências 12 14.

\section{Uma proposta didática}

As observações precedentes servem para que o professor possa entender as diferenças fundamentais entre as duas versões da lei de Hubble e a questão da expansão do universo. É fato que o limite de $z \ll 1$ da versão cosmológica da lei Hubble leva à lei de Hubble original, mas observe que nesse limite, poderemos deparar-nos com a forte influência dos movimentos próprios das galáxias que podem ofuscar completamente o movimento de expansão. Uma vez que o professor tenha esclarecido para si mesmo as nuances que envolvem a expansão do universo, ele poderá propor em sala de aula uma sequência de atividades que permitirão aos estudantes redescobrir a lei empírica de Hubble. Na nossa estimativa duas aulas de aproximadamente 50/55 minutos serão necessárias para desenvolver as atividades pertinentes.

\subsection{A proposta didática: introduzindo as ferramentas}

Na primeira das duas aulas o professor introduzirá o tópico da expansão do universo. Ao final da introdução ele deve formular a pergunta: como sabemos que o universo está em expansão? Para responder a esta pergunta o professor deverá convencer os alunos que é necessário antes introduzir alguns conceitos para que a resposta à pergunta inicial seja dada. Assim, tendo o professor motivado seus alunos, devem ser inicialmente abordados os tópicos:

(a) Noções básicas de espectroscopia; a influência do movimento da estrela sobre as linhas de emissão/ absorção. - ver Figura 2 da Seção 2 do presente trabalho. Como o deslocamento das linhas é descrito pelo efeito Doppler relativístico, convém que os alunos tenham uma idéia concreta deste efeito por meio do efeito Doppler sonoro na forma de uma demonstração rápida em sala de aula com um 'apitó eletrônico (tecnicamente: um buzzer piezolétrico) preso a uma corda e posto a girar. Nós utilizamos um buzzer de $2500 \mathrm{~Hz}$ com bons resultados.

(b) A seguir os alunos devem ser apresentados às equações do efeito Doppler relativistico, começando com a definição do parâmetro z. A Tabela 1 pode ser escrita no quadro-negro ou projetada sobre uma tela e as implicações devem ser discutidas.

Com exceção do item (b), estes tópicos estão alinhavados na Seção 2 do presente trabalho, ver também a Figura 2. A discussão deve ser qualitativa, isto é: a física envolvida deve ser enfatizada e deduções omitidas, somente as equações finais devem ser introduzidas. 
Tabela 1: Resumo do efeito Doppler relativístico. O deslocamento para o azul significa que a galáxia aproxima-se do observador; o deslocamento para o vermelho significa que esta afasta-se do observador.

\begin{tabular}{llcc}
\hline$z=\left(f_{\mathrm{E}}-f_{\mathrm{R}}\right) / f_{\mathrm{R}}$ & $z=\left(\lambda_{\mathrm{E}}-\lambda_{\mathrm{R}}\right) / \lambda_{\mathrm{R}}$ & vel. radial $(c z)$ & conclusão \\
\hline$f_{\mathrm{E}}<f_{\mathrm{R}} ; z<0$ & $\lambda_{\mathrm{R}}<\lambda_{\mathrm{E}} ; z<0$ & $V_{r}<0$ & desloc. $\mathrm{p} /$ o azul \\
\hline$f_{\mathrm{E}}>f_{\mathrm{R}} ; z>0$ & $\lambda_{\mathrm{E}}>\lambda_{\mathrm{R}} ; z>0$ & $V_{r}>0$ & desloc. $\mathrm{p} /$ o vermelho \\
\hline
\end{tabular}

\subsubsection{Reproduzindo Hubble 1929}

$\mathrm{Na}$ primeira parte da atividade, os alunos devem ser apresentados à Tabela 2 onde são mostrados os dados originais utilizados por Hubble no trabalho de 1929. A sequência didática para esta parte poderá ser:

(a) Apresentação/distribuição da Tabela 2 pelo professor com uma advertência especial à unidade de distância utilizada.

(b) O professor deve explicar o que significa fazer o ajuste linear, para isto pode fazer uso de uma simulação simples disponível no site do PhET:

https://phet.colorado.edu/pt_BR/simulation/ least-squares-regression

1. Os alunos devem digitar os dados correspondentes às distâncias e às velocidades mostradas na Tabela 2. Softwares gratuitos de regressão linear, como por exemplo, o Vernier Graphical Analysis ou QtiPlot podem ser facilmente obtidos na rede.

2. A pendente da reta deve ser obtida do gráfico gerado pelo software e comparado com o valor atual-

Tabela 2: Dados das 24 galáxias utilizados por Hubble [4]. As duas primeiras entradas correspondem à Pequena e a Grande Nuvem de Magalhões, as demais são galáxias listadas no New General Catalog (NGC).

\begin{tabular}{lcc} 
Galáxia (NGC) & Distância $(\mathrm{Mpc})$ & Vel. $(\mathrm{km} / \mathrm{s})$ \\
\hline S. Mag & 0,032 & 170 \\
\hline L. Mag & 0,034 & 290 \\
\hline 6822 & 0,214 & -130 \\
\hline 598 & 0,263 & -70 \\
\hline 221 & 0,275 & -185 \\
\hline 224 & 0,275 & -220 \\
\hline 5457 & 0,45 & 200 \\
\hline 4736 & 0,5 & 290 \\
\hline 5194 & 0,5 & 270 \\
\hline 4449 & 0,63 & 200 \\
\hline 4214 & 0,8 & 300 \\
\hline 3031 & 0,9 & -30 \\
\hline 3627 & 0,9 & 650 \\
\hline 4826 & 0,9 & 150 \\
\hline 5236 & 0,9 & 500 \\
\hline 1068 & 1,0 & 920 \\
\hline 5055 & 1,1 & 450 \\
\hline 7331 & 1,1 & 500 \\
\hline 4258 & 1,4 & 500 \\
\hline 4251 & 1,7 & 960 \\
\hline 4382 & 2,0 & 500 \\
\hline 4472 & 2,0 & 850 \\
\hline 4486 & 2,0 & 800 \\
\hline 4649 & 2,0 & 1090 \\
\hline & &
\end{tabular}

mente aceito. Lembrar aos alunos que a pendente tem unidades.

O gráfico resultante desta atividade é mostrado na Figura 7 , à esquerda.

\subsubsection{Reproduzindo Hubble com dados atualizados}

O procedimento descrito acima deve ser repetido com os dados de Hubble atualizados e disponíveis no banco de dados da NASA/IPAC: https://ned.ipac.caltech. edu Na Tabela 3 mostramos estes dados e a Figura 7, à direita, mostra o resultado do ajuste linear. Observe que a constante de Hubble obtida: $H_{0} \approx 72 \mathrm{~km} \mathrm{~s}^{-1} \mathrm{Mpc}^{-1}$ está razoavelmente em acordo com o valor atualmente aceito.

\section{Observações finais}

As propostas de atividades pedagógicas propostas aqui foram testadas no dia 26 de junho de 2018 em uma turma de nono ano do Ensino Fundamental no Colégio Tauá

Tabela 3: Dados atualizados das 24 galáxias utilizados por Hubble [4]. Como antes, as duas primeiras entradas correspondem à Pequena e á Grande Nuvem de Magalhões, as demais são galáxias listadas no New General Catalog (NGC). Observe a variação significativa nos valores das distâncias.

\begin{tabular}{|c|c|c|}
\hline Galáxia (NGC) & Distância (Mpc) & Vel. $(\mathrm{km} / \mathrm{s})$ \\
\hline S. Mag & 0,063 & 158 \\
\hline L. Mag & 0,050 & 278 \\
\hline 6822 & 0,520 & -77 \\
\hline 598 & 0,869 & -179 \\
\hline 221 & 0,768 & -200 \\
\hline 224 & 0,275 & -220 \\
\hline 5457 & 6,855 , & 241 \\
\hline 4736 & 5,107 & 308 \\
\hline 5194 & 7,185 & 463 \\
\hline 4449 & 3,864 & 207 \\
\hline 4214 & 2,979 & 291 \\
\hline 3031 & 3,674 & -34 \\
\hline 3627 & 9,592 & 727 \\
\hline 4826 & 5,405 & 408 \\
\hline 5236 & 6,422 & 513 \\
\hline 1068 & 10,582 & 1137 \\
\hline 5055 & 7,718 & 484 \\
\hline 7331 & 13,444 & 816 \\
\hline 4258 & 7,276 & 448 \\
\hline 4251 & 14,732 & 1066 \\
\hline 4382 & 15,243 & 729 \\
\hline 4472 & 16,031 & 981 \\
\hline 4486 & 16,747 & 1284 \\
\hline 4649 & 16,926 & 1110 \\
\hline
\end{tabular}



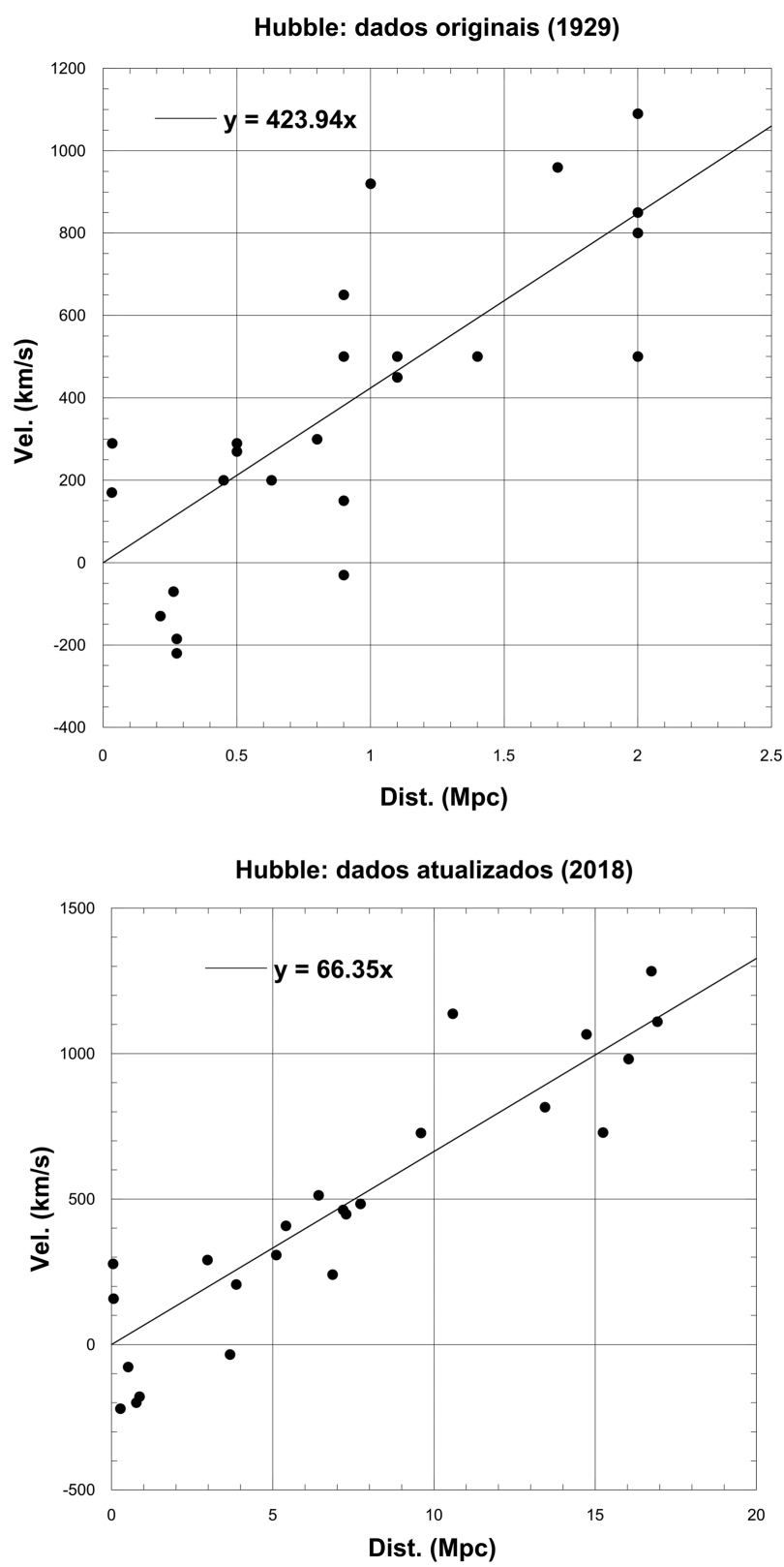

Figura 7: $\mathrm{O}$ primeiro gráfico foi obtido com os dados originais de Hubble utilizados no artigo de 1929. A constante de Hubble obtida vale $H_{0} \approx 403 \mathrm{~km} \mathrm{~s}^{-1} \mathrm{Mpc}^{-1}$, uma ordem de grandeza maior do que o valor aceito hoje em dia; as principais fontes de erro são as medidas de distância. O segundo gráfico foi obtido com as mesmas galáxias utilizadas por Hubble em 1929, mas com os dados de distância e velocidade atualizados. A constante de Hubble obtida é $H_{0} \approx 66 \mathrm{~km} \mathrm{~s}^{-1} \mathrm{Mpc}^{-1}$, razoavelmente próximo aos valor aceito atualmente. $\mathrm{O}$ ajuste linear segue $\mathrm{o}$ original proposto por Hubble, $y=m x$, ver [4 6].

(Ilha do Governador, Rio de Janeiro RJ) em dois tempos de aula de aproximadamente 50 minutos cada, com aproveitamento satisfatório. Acreditamos que a curiosidade que o tema (expansão do universo) provoca e a participação ativa que os alunos devem ter no decorrer das aulas sejam os principais motivos que nos levam a classificar o aproveitamento como satisfatório. No entanto, há es- paço para aperfeiçoamentos. É bem possível que em uma turma do Ensino Médio mais familiar com alguns dos conceitos introdutórios (ondas, efeito Doppler sonoro) para a qual este trabalho foi inicialmente pensado, o aproveitamento possa ser significativamente melhorado.

Finalmente, para o professor que desejar ilustrar a discussão sobre a expansão do universo, um modelo simples, mas esclarecedor é apresentado em [15]. Um apanhado geral e acessível sobre a cosmologia e seus rumos pode ser lido em 16.

\section{Agradecimentos}

Os autores agradecem ao árbitro pela leitura atenta do manuscrito original e por suas correções e sugestões. Um dos autores (V.R.P. da R.) agradece também a CAPES e à SBF e seu programa de Mestrado Nacional em Ensino de Física.

\section{Referências}

[1] J.D. Stein, Cosmic Numbers: The Numbers That Define Our Universe (Basic Books, Philadelphia, 2011).

[2] V.M. Slipher, Lowell Observatory Bulletin 2, 56 (1913).

[3] A.S. Eddington, The Mathematical Theory of Relativity (CUP, London, 1923).

[4] E. Hubble, Proc. Nat. Acad. Sci. 15, 168 (1929).

[5] E. Hubble, The Realm of the Nebulae (Yale University Press, New Haven, 2013).

[6] D.S. Soares e L.P.R. Soares, arXiv: 1312.0472v1 (2014).

[7] R.P. Kirshner, Proc. Nat. Acad. Sci. 101, 8 (2004).

[8] E. Hubble e M.L. Humason, Astrophys. J. 8043 (1931).

[9] A. Belenkly, Phys. Today 65, 38 (2012).

[10] H. Kragh e R.W. Smith, History of Science 41, 141 (2003).

[11] A. Bagdonas, J. Zanetic e I. Gurgel, Rev. Bras. Ens. Fís. 39, e2602 (2017).

[12] E. Harrison, Astrophys. J. 403, 28 (1993).

[13] E. Harrison Cosmology: The Science of the Universe (CUP, Cambridge, 2000) $2^{\mathrm{a}}$ ed.

[14] M.L. Bedran, Am. J. Phys. 70, 406 (2002).

[15] D. Soares, Rev. Bras. Ens. Fís. 36, 01 (2014).

[16] I. Waga, Rev. Bras. Ens. Fís. 27, 157 (2005). 